\title{
Genotyping in primary ciliary dyskinesia: ready for prime time, or a fringe benefit?
}

\author{
Claire Hogg, ${ }^{1}$ Andrew Bush ${ }^{1,2}$
}

Once upon a time, many diagnoses were easy-for example, if you had a high sweat chloride, you had cystic fibrosis (CF); if the sweat electrolytes were normal, you did not. Now, recent advances in molecular genetics and airway electrophysiology have pushed back and blurred the diagnosis of $\mathrm{CF}$, such that what was once straightforward can in atypical cases become the source of endless debate. The same has become true in primary ciliary dyskinesia (PCD). The situation in PCD is even more complicated; diagnosis seldom relied on a single test, but has become confusing because of the large number of tests currently available, many of which are very sophisticated and only available in a small number of centres. So, what is a PCD diagnosis in the 21 st century?

First, of course, is it necessary to make a specific diagnosis of PCD at all? There are several reasons why it is; the treatment of upper airway disease in PCD is counterintuitive $^{1}$ and there are genetic implications both for PCD and other parts of the expanding ciliopathy spectrum for the families. Importantly, there are no big randomised controlled trials of treatment in PCD; most treatments are extrapolated from protocols used for $\mathrm{CF}$, a disease with completely different pathophysiology (reduced airway surface liquid rather than primary ciliary dysmotility). So, if this deficiency is to be addressed, the accurate diagnosis of PCD is a prerequisite. The same is also true for studies in basic mechanisms, including finding novel PCD genes.

There is wide variation in availability of the different tests across Europe ${ }^{2}$; the

\footnotetext{
${ }^{1}$ Paediatric Respiratory Department, Royal Brompton Hospital, Sydney Street, London, UK; ${ }^{2}$ Paediatric Respiratory Department, Imperial College, South Kensington Campus, London, UK

Correspondence to Dr Claire Hogg, Paediatric Respiratory Department, Royal Brompton Hospital, Sydney Street, Chelsea, London SW3 6NP, UK; c.hogg@rbht.nhs.uk
}

range is summarised in table 1 . There is no one single clinical feature which can be taken as diagnostic of PCD, although many should prompt consideration of the diagnosis. Thus, although it is totally legitimate for clinicians without access to diagnostic tests to make a clinical presumptive diagnosis of PCD and treat the patient accordingly, we do not believe that patients with a clinical diagnosis alone should ever be entered into clinical trials or studies of basic mechanisms; some supportive evidence is essential. We suggest from the evidence summarised in table 1 that PCD is only reliably excluded by a normal ciliary beat pattern and frequency. Abnormalities of ciliary beat pattern are the most consistent diagnostic feature in every large cohort of patients, where evidence suggests that up to $20 \%$ of

patients have normal electron microscopy $^{8}$ and, where defined, around 40\% of patients have two known genetic mutations in trans. ${ }^{9}$ Beat pattern in experienced hands has become a more useful tool than ciliary beat frequency, which may be normal in patients with an abnormal beat pattern and a definite ultrastructural defect on electron microscopy. ${ }^{10}$ Conversely, a disease-producing ultrastructural defect with a normal beat pattern has never been convincingly reported. For these reasons, ciliary function analysis using light microscopy and fast video technology should be at the forefront of any diagnostic set-up. A normal nasal nitric oxide should cast strong doubt on the diagnosis. PCD is definitely diagnosed if (a) there is a repeatedly abnormal ciliary beat pattern and/or frequency on an adequate sample of epithelial cells (with ciliary culture if there is any doubt); or (b) electron microscopy reveals a definite diseasecausing abnormality such as an outer dynein arm defect; or (c) genetic studies reveal two known disease-producing mutations in trans. All other combinations of tests can only diagnose 'probable PCD', and clinicians and investigators are left to determine what degree of probability is acceptable in that particular context. Specifically, it is incorrect to consider

Table 1 Diagnostic testing in primary cell dyskinesia (PCD)

\begin{tabular}{lll}
\hline Test & Interpretation & Role in PCD diagnosis \\
\hline Saccharin test $^{3}$ & $\begin{array}{l}\text { False negatives and false positives, } \\
\text { screening test at best }\end{array}$ & Not useful to diagnose or exclude PCD \\
$\begin{array}{l}\text { Radionuclide mucociliary } \\
\text { clearance }^{4}\end{array}$ & $\begin{array}{l}\text { Good sensitivity and specificity but } \\
\text { only limited experience or access in }\end{array}$ & Not useful to diagnose or exclude PCD
\end{tabular}

Nasal nitric oxide ${ }^{5}$

Ciliary motility studies

Transmission electron microscopy

Culture of ciliary biopsy ${ }^{6}$

Genetic studies

Immunofluorescence of ciliary proteins ${ }^{7}$ most centres

Used as the screening test of choice, also low in cystic fibrosis and other conditions; now also part of the diagnostic investigation

Can be affected by recent viral infection

Can be affected by recent viral infection

Cilia regrown in culture, used to differentiate secondary ciliary dyskinesia in PCD

$>250$ potential loci, very few known genes

Limited experience only and applicable to date to very few proteins
Normal nasal nitric oxide $=$ PCD very unlikely

Normal ciliary beat frequency and pattern = PCD excluded If abnormal, repeat after treatment of infection or proceed to ciliary culture Abnormal ciliary beat frequency and/or pattern on an adequate sample = definite PCD

Presence of known disease producing structural abnormality $=$ definite PCD Can be normal with definite PCD Normal ciliary beat frequency and pattern $=$ PCD excluded Abnormal ciliary beat frequency and/or pattern on an adequate sample $=$ definite PCD

Two known disease producing mutations in trans = definite PCD Can be no known mutations detected with definite PCD

Insufficient evidence to evaluate a role in clinical practice

Not a stand-alone diagnostic test 
electron microscopy in isolation as the 'gold standard', no matter how skilled the operator.

In their paper published in this issue of Thorax, Knowles et al (see page 433) report on the sequencing of 82 exons and intron/exon junctions in DNAH11 in 163 patients with a 'clinical phenotype' of PCD, including 58 with normal ciliary ultrastructure. $^{11}$ Thirteen of this latter group had biallelic mutations in the gene as well as two patients with PCD in whom ultrastructural analysis had not been performed. No patients with conventional ultrastructural abnormalities causing PCD had biallelic mutations, nor did those with isolated situs inversus. This carefully obtained body of data leads to two questions: (1) Did the patients they studied with normal ultrastructure in fact have PCD? (2) Where do their data play into the diagnostic pathway for PCD?

As discussed above, the diagnosis of PCD may be very complex. It is clear that at least some of the patients in the study by Knowles et al either did not have PCD at all or had a questionable diagnosis. Situs inversus with no symptoms and a normal nasal nitric oxide is just not PCD, whatever mouse data may say. Few would feel completely confident about diagnosing PCD in a patient with no neonatal respiratory distress, no measurement of nasal nitric oxide and only bronchiectasis and situs inversus. Isolated situs inversus is well described and does not give exemption from bronchiectasis due to other causes. Indeed, 23 patients did not have formal ciliary ultrastructural studies at all. Nonetheless, despite these caveats, the authors have made a compelling case for the importance of DNAH11 mutations in PCD with normal ciliary ultrastructure, not least because of their demonstration of the effects of the mutations on DNA transcripts. However, I suggest that it would be important to tighten up the diagnostic criteria for future studies.

What then are the implications of their data for PCD diagnosis? Clearly, their data confirm that electron microscopy can no longer be considered the gold standard diagnosis for PCD and centres that rely purely on this technique will miss many diagnoses. With regard to the place of genetic tests in the diagnostic pathway, perhaps a comparison with a genetically much simpler disease (ie, CF) is instructive. $\mathrm{CF}$ is a monogenic disorder with $>1800$ possibly disease-producing mutations described, although in fact $<50$ meet rigid criteria as disease-producing. ${ }^{12}$ Even in this genetically simpler disease, a functional test-in this case, sweat chloride-is recommended to be the primary diagnostic test. We believe that this should be the case in PCD, not least because of the vastly greater number of genetic loci and the far lesser success rate of genetic diagnosis. It is simply not good enough to dismiss videomicroscopy as 'difficult and limited in availability'; if it is the best test, it should be made available. No-one would advocate abandoning the sweat test because unskilled use leads to false positives and negatives, nor should functional ciliary studies be displaced because they are not easy and the equipment is sophisticated.

So, in summary, where in the diagnostic process for PCD should genetic tests be positioned? For current clinical (as opposed to research) purposes, the diagnosis of PCD rests on nasal nitric oxide, ciliary beat frequency and pattern, and electron microscopy. In the relatively few patients in whom these tests are equivocal, there are other tests available including ciliary culture, immunofluorescence of ciliary proteins and genetic testing. Genetic testing can certainly be useful diagnostically if the standard tests are equivocal, but it should be noted that all of the patients found by Knowles et al to have DNAH11 mutations in whom ciliary functional studies were also performed had a dyskinetic beat pattern and thus could have been diagnosed without recourse to gene sequencing. Genetic testing may be useful if an antenatal diagnosis is sought by families with a child with PCD who has a known genotype and in the future when genotype-specific therapies are explored in PCD (eg, ataluren in nonsense mutations) but, at the moment, the answer to the question we set ourselves is 'fringe benefit' for most patients in the PCD diagnostic pathway. Groups setting up to diagnose PCD should be concentrating on making really good ciliary functional measurements, exchanging video clips with other centres for quality control as a prerequisite for present diagnosis and future sophisticated studies of the molecular biology of PCD rather than obsessing on (with apologies to Peter Cook and Dudley Moore) 'Beyond the Fringe'!

\section{Competing interests None.}

Provenance and peer review Commissioned; internally peer reviewed.

Published Online First 9 January 2012

Thorax 2012:67:377-378

doi:10.1136/thoraxjnl-2011-201320

\section{REFERENCES}

1. Bush A, Chodhari R, Collins N, et al. Primary ciliary dyskinesia: current state of the art. Arch Dis Child 2007;92:1136-40.

2. Barbato A, Frischer T, Kuenhi CE, et al. Primary ciliary dyskinesia: a consensus statement on diagnostic and treatment approaches in children. Eur Respir J 2009;34:1264-76.

3. Bush A, Cole P, Hariri M, et al. Primary ciliary dyskinesia: diagnosis and standards of care. Eur Respir J 1998;12:982-8.

4. De Boeck K, Proesmans M, Mortelmans L, et al. Mucociliary transport using $99 \mathrm{~m}$ Tc-albumin colloid: a reliable screening test for PCD. Thorax 2005;60:414-17.

5. Leigh MW, Zariwala MA, Knowles MR. Primary ciliary dyskinesia: improving the diagnostic approach Curr Opin Pediatr 2009;21:320-5.

6. Hirst RA, Rutman A, Williams G, et al. Ciliated air-liquid cultures as an aid to diagnostic testing for primary ciliary dyskinesia. Chest

2010;138:1441-7.

7. Fleigauf $\mathbf{M}$, Obrich $\mathrm{H}$, Harvath $\mathrm{J}$, et al. Mislocalisation of DNAH5 and DNAH9 in respiratory cells from patients with primary ciliary dyskinesia. Am J Respir Crit Care Med 2005;171:1343-9

8. Zariwala MA, Knowles MR, Omran H. Genetic defects in ciliary structure and function. Annu Rev Physiol 2007;69:423-50.

9. Hornef $\mathbf{N}$, Olbrich $\mathrm{H}$, Horvath J, et al. DNAH5 mutations are a common cause of primary ciliary dyskinesia with outer dynein arm defects. Am J Respir Crit Care Med 2006;174:120-6.

10. Chilvers MA, Rutman A, O'Callaghan C. Primary ciliary dyskinesia: ciliary beat pattern is associated with specific ultrastructural defects. J Allergy Clin Immunol 2003;112:518-24.

11. Knowles MR, Leigh M, Carson J, et al. Mutations of DNAH11 in primary ciliary dyskinesia patients with normal ciliary ultrastructure. Thorax 2012;67:433-41.

12. Castellani C, Cuppens H, Macek M Jr, et al. Consensus on the use and interpretation of cystic fibrosis mutation analysis in clinical practice. J Cyst Fibros 2008:7:179-96. 